\title{
Preparação e Caracterização de Compósitos Poliméricos Baseados em Amido Termoplástico e Materiais de Alta Área Superficial: Zeólita ZSM-5 e Sílica Coloidal
}

\author{
Fábio Plotegher \\ Programa de Pós Graduação em Química, UFSCar
}

\author{
Caue Ribeiro \\ EMBRAPA Instrumentação
}

Resumo: Foram produzidas amostras de amido termoplástico (TPS) reforçadas com materiais de alta área superficial, com o intuito de verificar a variação nas propriedades do polímero, com especial enfoque na sua permeabilidade a vapor d'água. Foram utilizadas como carga a sílica coloidal (área superficial de $122,7 \mathrm{~m}^{2} / \mathrm{g}$ ) e uma zeólita do tipo ZSM-5, produzida em laboratório (área superficial de $261,3 \mathrm{~m} 2 / \mathrm{g}$ ), em teores de 2 a $10 \%$ em massa. Os resultados demonstraram que a adição de ambos os materiais melhorou as propriedades mecânicas do TPS, embora nos maiores teores houve redução da qualidade das interfaces e dessas propriedades, principalmente para a ZSM-5. Em todos os casos a introdução da carga inorgânica reduziu a permeabilidade ao vapor d'água em até $20 \%$ quando comparada à permeabilidade do TPS, porém a melhor dispersão da sílica coloidal na matriz permitiu as maiores reduções, apesar da área superficial inferior.

Palavras-chave: Compósito, amido termoplástico, TPS, ZSM-5, zeólita, sílica coloidal.

\section{Preparation and Characterization of Polymeric Composites Based on Thermoplastic Starch and High Surface Area Materials: ZSM-5 Zeolite and Colloidal Silica}

\begin{abstract}
Compositions of thermoplastic starch (TPS) reinforced by high surface area materials were produced, intending to study the variation in polymer properties, focusing on the permeability to water vapor. Colloidal silica (surface area $122.7 \mathrm{~m}^{2} / \mathrm{g}$ ) and a ZSM-5 zeolite (surface area $261.3 \mathrm{~m}^{2} / \mathrm{g}$ ) were used, in loadings from 2 to $10 \%$ weight. The results demonstrated that the addition of both materials was favorable to the TPS mechanical properties, however in higher loadings the quality of polymer interfaces and these properties were negatively affected, especially for ZSM-5. In all the cases the inorganic particles reduced the permeability to water vapor in levels below $20 \%$ when compared to pure TPS, although the best dispersion of colloidal silica determined better reductions, despite its lower surface area.
\end{abstract} Keywords: Composite, thermoplastic starch, TPS, ZSM-5, zeolite, colloidal silica.

\section{Introdução}

Atualmente, vários trabalhos de pesquisa estão focados em sistemas poliméricos biodegradáveis como alternativas ambientalmente corretas e adequadas para produção de embalagens, pelo seu rápido descarte. Muitos polímeros biodegradáveis provenientes da biomassa vêm sendo estudados, especialmente os polímeros naturais baseados em precursores polissacarídicos $^{[1]}$, tais como a celulose e seus derivados ${ }^{[2]}$, a quitina e quitosana ${ }^{[3]}$, e o amido termoplástico ${ }^{[4,5]}$. Este último, também conhecido na literatura como TPS, tem destaque por sua facilidade de processamento em condições típicas de polímeros em geral, permitindo a produção de filmes, injetados e expandidos ${ }^{[6]}$.

No entanto, apesar destes materiais permitirem o descarte direto, a sua origem natural muitas vezes dificulta a padronização de propriedades como resistência mecânica e permeabilidade a gases, sendo freqüentes os problemas relacionados à reprodutibilidade. Uma forma de minimizar esses problemas é a utilização destes polímeros como compósitos ${ }^{[7-9]}$, que são considerados todos os materiais nos quais duas ou mais fases de origens distintas coexistem em escala pelo menos micrométrica, com interfaces bem definidas. Desta forma, a seleção de cargas adequadas para modificar o comportamento do polímero matriz é uma etapa fundamental do projeto de produtos adequados. Para o TPS, algumas cargas como fibras naturai ${ }^{[10]}$, nanofibras de celulose, nanotubos de carbono, sílica nanométrica ${ }^{[11,12]}$, e $\operatorname{argilas}^{[13]}$ mostraram bons ganhos com relação à resistência mecânica do material.
Porém, até o momento, poucos dados foram estabelecidos para o comportamento das demais propriedades do TPS modificado com cargas inorgânicas. Especificamente quanto à produção de filmes de TPS, um dos fatores de maior importância é a padronização da sua permeabilidade a gases e, especificamente, ao vapor d'água, considerando-se aplicações na área de embalagens de alimentos. Assim, materiais potencialmente interessantes para uso como cargas seriam estruturas adsorventes de alta área de superfície, tais como zeólitas e sílica coloidal. Deve-se notar que ambas as cargas foram pouco estudadas sobre seu efeito na permeabilidade de filmes de TPS e, inclusive, estudos sobre incorporação de zeólitas em TPS ainda não estão disponíveis na literatura.

Desta forma, este trabalho descreve a preparação e caracterização de compósitos a partir de TPS e dois sistemas diferentes de alta área superficial: uma zeólita ZSM-5, preparada em laboratório e sílica coloidal comercial. Pretende-se assim contribuir com o conhecimento sobre as interfaces nestes sistemas e adicionalmente, propor alternativas para o uso deste conhecimento em polímeros biodegradáveis.

\section{Material e Métodos}

A zeólita escolhida para este trabalho foi uma ZSM-5, conhecida por seu comportamento de alta absorção de gases, em procedimento modificado a partir de condições anteriormente descritas $^{[14-16]}$. A estrutura cristalina da zeólita foi comprovada

Autor para correspondência: Caue Ribeiro, EMBRAPA Instrumentação, Rua XV de Novembro, 1452, São Carlos, SP, CEP 13561-206,

e-mail: caue.ribeiro@embrapa.br 
através de análises de difração de raios $X$, realizadas em um difratômetro Shimadzu modelo LabX XRD-6000, operado com radiação $\mathrm{Cu}-\mathrm{k} \alpha$ de $\lambda=1,54056 \AA$, tensão de $30 \mathrm{kV}$, corrente de 30 $\mathrm{mA}$, com velocidade contínua de $0,5^{\circ}(2 \theta) / \mathrm{min}$, com variação do ângulo na faixa de 5 a $35^{\circ}(2 \theta)$. A área superficial relativa foi obtida por meio da técnica de fissisorção de nitrogênio com isoterma de BET em um equipamento ASAP modelo 2020 da Micromeritics Corporation. A morfologia das partículas foi identificada por microscopia eletrônica de varredura, visualizadas em um microscópio eletrônico Zeiss ${ }^{\circledR}$ modelo Supra 35 FEG, operado com tensão de $3 \mathrm{kV}$, utilizando o detector de elétrons secundário.

A sílica coloidal comercial empregada no trabalho foi a Tixosil $333^{\circledR}$ da Rhodia, e foi caracterizada por fisissorção de nitrogênio e sua morfologia por microscopia eletrônica de varredura, em um microscópio SEM-FEG da JEOL ${ }^{\circledR}$ modelo JSM 6701F.

As misturas de TPS foram realizadas segundo condições padrão já conhecidas na literatura ${ }^{[6,17]}$. A matriz foi produzida contendo $30 \%$ de glicerol (Synth 98\%), 1\% de ácido esteárico (Synth 98\%), $69 \%$ de amido (Amidex ${ }^{\circledR}$ 3001). Foram feitas misturas adicionando 0, 2, 4, 6, 8 e $10 \%$ em massa do reforço. Para o processamento, foi utilizado um misturador de câmara interna HAAKE Polylab OS Rheodrive 4 da Thermo Electron Corporation, com rotor tipo Roller, a $160{ }^{\circ} \mathrm{C}, 200 \mathrm{rpm}$ durante 5 minutos ${ }^{[17]}$. Após o processamento, o material foi moldado como filmes em termoprensa, por pré aquecimento do material por 6 minutos a $160{ }^{\circ} \mathrm{C}$, prensagem de 5 toneladas por 2 minutos e nova prensagem de 10 toneladas por mais 2 minutos.

As composições foram caracterizadas por análise termogravimétrica (TGA) em um equipamento TA Instruments ${ }^{\circledR}$ modelo TGA-Q500, com rampa de aquecimento de $10^{\circ} \mathrm{C} / \mathrm{min}$ até $700{ }^{\circ} \mathrm{C}$ sob atmosfera oxidante de ar sintético com fluxo de $60 \mathrm{~mL} / \mathrm{min}$. As análises térmicas dinâmico-mecânica (DMTA) foram realizadas em um equipamento TA Instruments ${ }^{\circledR}$ modelo DMA-Q800 utilizando geometria retangular "Dual Cantilever", com deformação senoidal no modo flexão de três pontos com condições padronizadas de perturbação senoidal de $1 \mathrm{~Hz}$ de freqüência, amplitude de $20 \mu \mathrm{m}$ e taxa de aquecimento de $2{ }^{\circ} \mathrm{C} / \mathrm{min}$, variando de $-120^{\circ} \mathrm{C}$ até $150{ }^{\circ} \mathrm{C}$.

A fratura a frio com nitrogênio líquido e a composição química dos corpos de prova foram caracterizadas por meio de microscopia eletrônica de varredura com detector de energia dispersiva de raios $\mathrm{X}$ em um equipamento SEM-EDS da JEOL ${ }^{\circledR}$ modelo JSM 6510.

Os ensaios de resistência mecânica à tração foram feitos segundo norma internacional ASTM D 638-08 (materiais tipo II). Empregou-se uma máquina EMIC DL 3000 com célula de carga de $50 \mathrm{kgf}$ e velocidade de $50 \mathrm{~mm} / \mathrm{min}$ até a fratura do material.

$\mathrm{O}$ teste de permeabilidade a vapor d'água foi feito por meio do método gravimétrico estático adaptado da norma internacional ${ }^{[13,18,19]}$. O sistema foi montado com $3 \mathrm{~g}$ de sílica gel ativada $\left(200{ }^{\circ} \mathrm{C}\right)$ seca dentro de um frasco de $7 \mathrm{~mL}$, selados hermeticamente com as amostras dos filmes preparados. Os sistemas foram acondicionados em dessecador contendo solução saturada de cloreto de sódio $(\mathrm{NaCl}$, Synth $98 \%)$, para promover umidade relativa de $75 \% \pm 3$, monitorada por um Termo-higrômetro Minipa MT 240. O ganho de massa de cada sistema foi monitorado por meio de uma balança analítica com precisão de $0,00001 \mathrm{~g}$ a cada 24 horas por 27 dias. A partir dos dados coletados, foram construídos gráficos de variação de massa em função do tempo (g/dia). A partir da região linear de cada curva foi obtido o coeficiente linear que taxa de transmissão de vapor d'água (WVT) corrigido pela área $\left(\mathrm{A} \mathrm{em} \mathrm{m}^{2}\right)$ de transferência disponível para o transporte de vapor d'água, como mostra a Equação 1:

$$
\mathrm{WVT}=(\Delta \mathrm{m} / \Delta \mathrm{t}) / \mathrm{A}
$$

Onde $\Delta \mathrm{m}$, corresponde à variação da massa em gramas e $\Delta \mathrm{t}$, o tempo em dias. Aplica-se a $1^{\text {a }}$ Lei de Fick, para determinar o coeficiente de permeabilidade ao vapor d'água, $\mathrm{Pw}$ (g/Pa.dia.m), através da Equação 2:

$$
\mathrm{Pw}=[\mathrm{WVT} / \mathrm{S}(\mathrm{R} 1-\mathrm{R} 2)] . \mathrm{x}
$$

Onde S é a pressão de saturação do vapor d'água (Pa), na temperatura que foi executada o teste, R1, é a umidade relativa do dessecador $(75 \% \pm 3), \mathrm{R} 2$, a umidade relativa dentro da célula do sistema $(0 \%)$ e x é a espessura do filme em metros (m).

\section{Resultados e Discussão}

A Figura 1 mostra o difratograma de raios $\mathrm{X}$ da zeólita obtida. Pode-se comprovar que a fase presente corresponde à zeólita ZSM-5, e não foram observadas outras fases residuais ${ }^{[20,21]}$. Cabe destacar que o processo foi otimizado para obtenção da fase em menores tempos do que as condições propostas na literatura ${ }^{[14,15]}$. Estudos preliminares comprovam que temperaturas muito baixas influenciam o processo de nucleação de formação do cristal $^{[22]}$, porém, mesmo assim, é possível a obtenção da fase zeolítica a $70^{\circ} \mathrm{C}$, por exemplo ${ }^{[15]}$. Porém, o tempo de síntese passa a ser muito longo, chegando a passar dos 15 dias de tratamento hidrotérmico ${ }^{[15,23-25]}$.

A Figura 2a mostra a micrografia da zeólita ZSM-5 sintetizada. Observa-se um aglomerado formado por partículas de cristais ortorrômbicos bem definidos característicos desse tipo de zeólita. A Figura 2b mostra um aglomerado de partículas de sílica coloidal, onde observa-se que as partículas são bem menores que as partículas da zeólita sintetizada, e apresentam-se aglomeradas. Pelas imagens não é possível determinar se os aglomerados observados, tanto da ZSM-5 quanto da sílica coloidal, são frágeis e poderão ser dispersos pela ação mecânica no processo de mistura com a matriz polimérica. No entanto, os resultados obtidos nas análises de fisissorção de nitrogênio mostraram para ambos os sistemas altos valores de área superficial, que indicam a presença de partículas com poucos pontos de contato, preservando-se a acessibilidade de área. Para a zeólita os valores médios foram de $261,3 \mathrm{~m}^{2} / \mathrm{g}$, já para a sílica os valores foram de $122,7 \mathrm{~m}^{2} / \mathrm{g}$. Apesar de a zeólita apresentar partículas muito maiores que as partículas de sílica, sua área superficial maior é explicada pela própria estrutura interna da zeólita, que possui poros e canais sinusoidais bidimensionais, que quando desobstruídos pela calcinação revelam uma área superficial até então inacessível.

O processamento das composições de TPS com ambas as estruturas escolhidas mostrou-se mais eficiente a cada aumento de carga incorporada. Observou-se que a massa do compósito obtida no processamento soltava-se mais facilmente dos rotores e da parede da câmara de mistura. Os corpos de prova foram produzidos

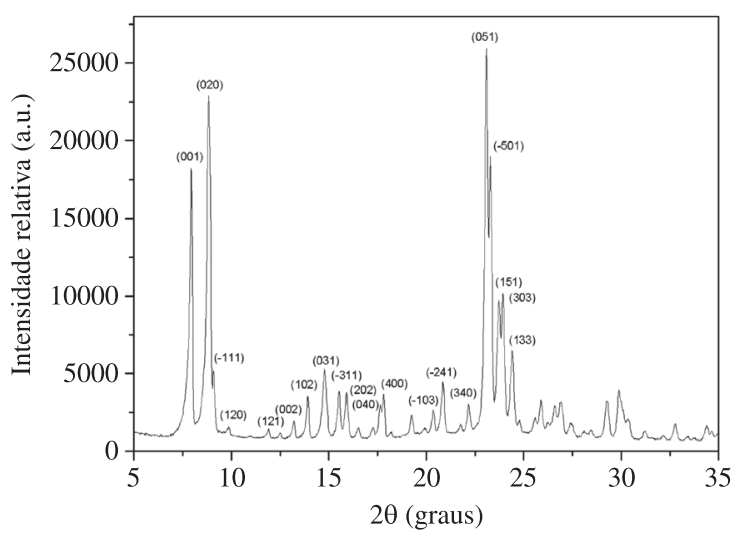

Figura 1. Difratograma de raios X da zeólita ZSM-5. 
como filmes com relativa transparência, como visto na Figura 3, e acondicionados à umidade controlada de $53 \%$ para os ensaios subsequentes ${ }^{[26]}$.

As análises termogravimétricas de todas as composições mantiveram o sempre o mesmo perfil de perda de massa comparada ao perfil do TPS puro, indicando que a presença das ambas as cargas não alterou comportamento de perda de massa, como mostrado na Figura 4. Observando os gráficos das perdas de massa nota-se que o processo ocorre em três regiões principais: a primeira corresponde à perda de umidade existente no material $\left(<200^{\circ} \mathrm{C}\right)$, a segunda etapa corresponde à decomposição e despolimerização das cadeias de amilose e amilopectina componentes do amido (250 a $320{ }^{\circ} \mathrm{C}$ ) e a terceira etapa é a eliminação do material carbonáceo pela formação de monóxido e/ou dióxido de carbono $\left(>450{ }^{\circ} \mathrm{C}\right)$.

Os dados de termogravimetria sugerem que a interação do TPS com as cargas inorgânicas é baixa, porém, a análise dinâmico-mecânica, apresentada na Figura 5, sugere interação em baixas temperaturas, ou seja, em condições nas quais o polímero

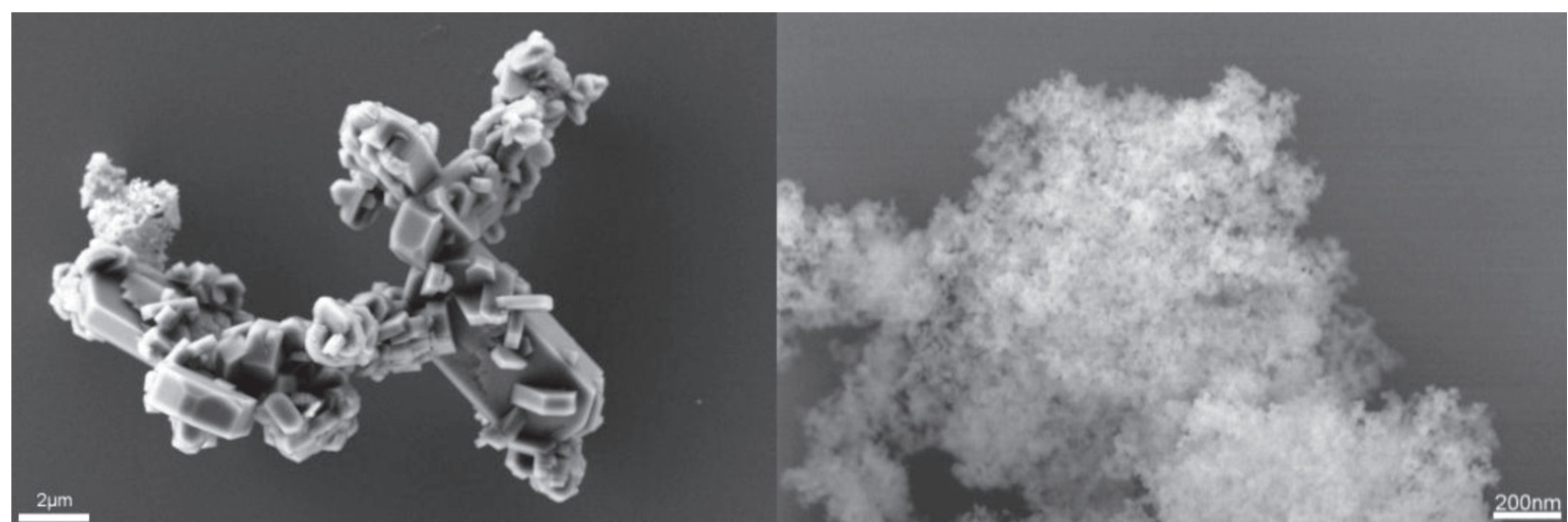

(a)

(b)

Figura 2. Micrografia da zeólita ZSM-5 (a) e sílica comercial Tixosil $333^{\circledR}$ (b).

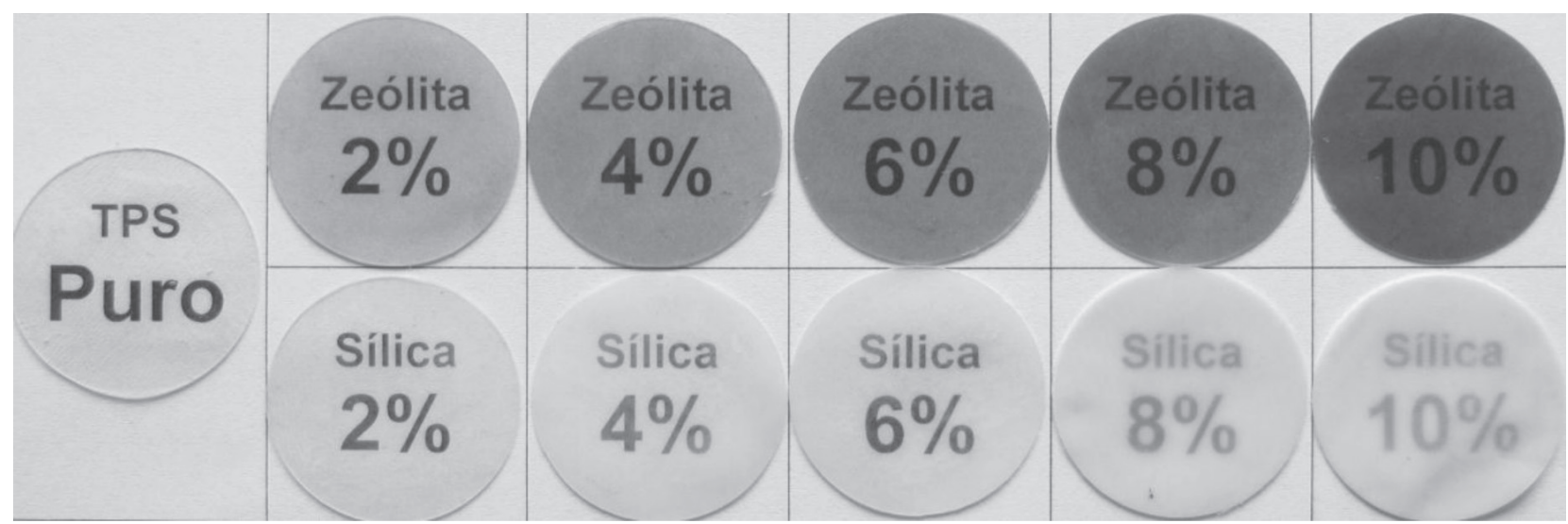

Figura 3. Transparência dos filmes para o teste de permeabilidade ao vapor d'água.
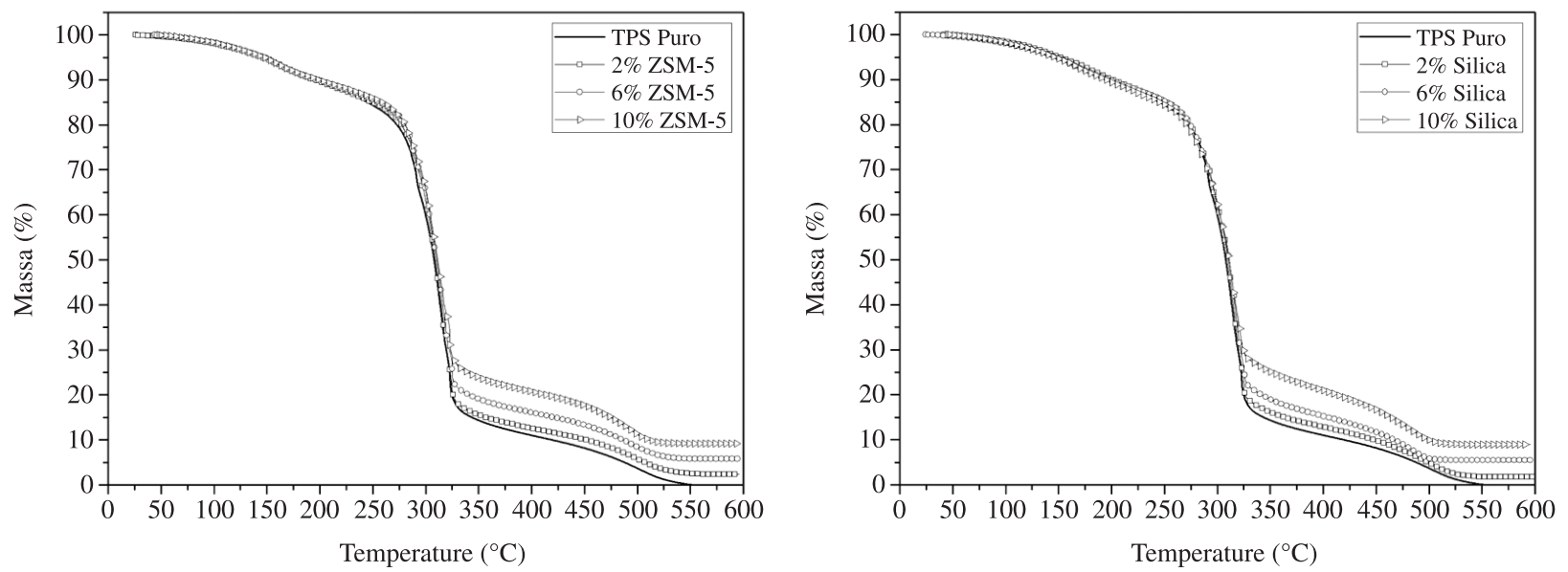

Figura 4. Análise termogravimétrica das composições. 
apresenta-se rígido. Nota-se nesse experimento o aumento no módulo de estocagem das curvas para cada aumento da carga inorgânica. De fato, espera-se que haja interação entre o polímero e ambas as cargas, visto que são todos sistemas hidrofílicos e, portanto, não seriam necessários agentes de compatibilização nestes sistemas. As curvas de tangente de perda $(\tan \delta$ ) apresentadas na Figura 5 exibem dois picos distintos: de acordo com os estudos de Avérous et al. ${ }^{[27]}$, o primeiro pico em entre $-55 \mathrm{e}-60{ }^{\circ} \mathrm{C}$, corresponde à $\mathrm{Tg}$ do glicerol ou de fases ricas em glicerol. $\mathrm{O}$ segundo pico mais largo, entre 0 e $20^{\circ} \mathrm{C}$, corresponde à transição vítrea de fases ricas em amido. Cabe destacar que nas curvas tan $\delta$ para o sistema TPS/sílica, as temperaturas de transição vítrea do glicerol e amido foram deslocadas para temperaturas mais altas com relação à curva do TPS puro. Esse comportamento provavelmente está associado ao fato das partículas de sílica coloidal serem muito pequenas (Figura $2 b$ ) podendo estar melhor dispersas na matriz polimérica que no sistema TPS/zeólita, e ocupando os vazios que acabam influenciando o movimento das cadeias poliméricas de amilose e amilopectina do amido.

Os valores coletados das curvas dos ensaios de tração mecânica utilizadas no tratamento estatístico para a diferenciação das composições foram: tensão $(\sigma)$ e deformação $(\varepsilon)$ de ruptura e principalmente o módulo elástico $(\mathrm{E})$. A Tabela 1 mostra as médias obtidas no ensaio mecânico. Observou-se que a presença de ambas as cargas foi favorável ao aumento da resistência mecânica, tanto do aumento do módulo de Young quanto da tensão de ruptura dos compósitos produzidos. A queda na elongação somente foi sensível, para ambos os casos, nos teores máximos (8 e 10\% de carga).

No entanto, deve-se notar que, em teores de $6 \%$ de ZSM-5, o comportamento mecânico apresentou queda, enquanto nas adições de sílica coloidal houve aumento das propriedades continuamente com

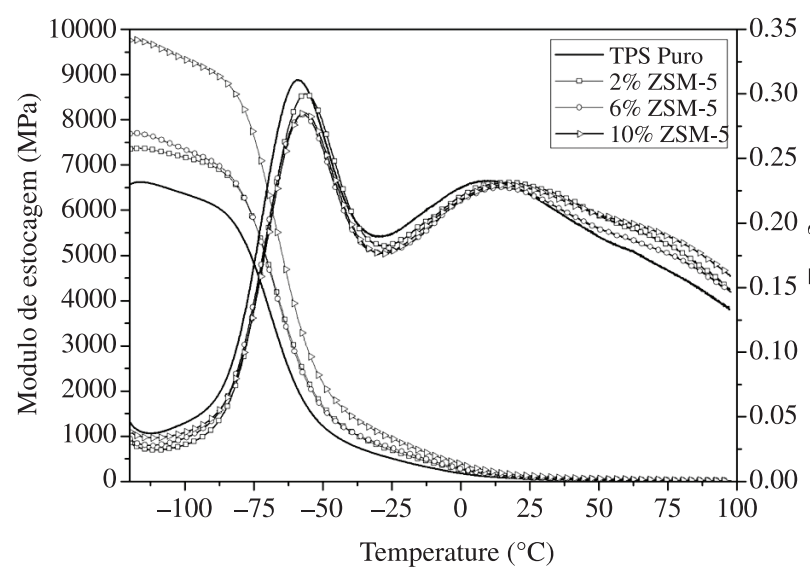

Figura 5. Curvas de módulo de estocagem e Tan $\delta$ das composições.

Tabela 1. Valores médios do ensaio mecânico.

\begin{tabular}{lccc}
\hline Composição & Módulo elástico $(\mathbf{E})$ MPa & Tensão ruptura $(\sigma)$ MPa & Deformação de ruptura $(\varepsilon)$ \% \\
\hline TPS Puro & $10,14 \pm 2,73$ & $0,84 \pm 0,18$ & $0,71 \pm 0,19$ \\
TPS + 2\% Sílica & $12,98 \pm 3,17$ & $0,77 \pm 0,20$ & $0,64 \pm 0,13$ \\
TPS + 4\% Sílica & $23,70 \pm 8,03$ & $1,28 \pm 0,17$ & $0,70 \pm 0,09$ \\
TPS + 6\% Sílica & $33,74 \pm 12,09$ & $1,55 \pm 0,41$ & $0,46 \pm 0,05$ \\
TPS + 8\% Sílica & $31,99 \pm 15,48$ & $1,26 \pm 0,33$ & $0,44 \pm 0,06$ \\
TPS + 10\% Sílica & $36,09 \pm 16,46$ & $1,28 \pm 0,42$ & $0,36 \pm 0,06$ \\
TPS + 2\% ZSM-5 & $21,08 \pm 5,21$ & $1,15 \pm 0,22$ & $0,52 \pm 0,09$ \\
TPS + 4\% ZSM-5 & $37,72 \pm 9,95$ & $1,58 \pm 0,25$ & $0,60 \pm 0,12$ \\
TPS + 6\% ZSM-5 & $24,03 \pm 6,09$ & $1,15 \pm 0,17$ & $0,60 \pm 0,14$ \\
TPS + 8\% ZSM-5 & $24,76 \pm 7,08$ & $1,12 \pm 0,17$ & $0,69 \pm 0,12$ \\
TPS + 10\% ZSM-5 & $26,65 \pm 11,59$ & $1,28 \pm 0,33$ & $0,56 \pm 0,10$
\end{tabular}

o maior carregamento. Esse fato pode novamente ser explicado pela possível melhor dispersão das partículas de sílica coloidal durante o processamento, reduzindo falhas críticas como destacamentos ou rupturas na interface. De fato, este comportamento é verificado na Figura 6, onde são vistas as secções transversais dos corpos de prova, fraturados criogenicamente, em que é observada a presença mais extensa de falhas na interface carga / polímero. Nota-se que em menores teores a interação é melhor, evidenciada pela boa qualidade da interface nestas condições.

A Tabela 2 mostra os resultados obtidos do experimento de permeabilidade ao vapor d'água. Nota-se que a adição de ambas as cargas reduziu em até $20 \%$ a permeabilidade a vapor d'água dos compósitos, porém, em maiores adições o efeito de redução é diminuído. Novamente, pode-se atribuir essa redução da propriedade pelo aumento das falhas de interface, como visto na Figura 6 para ambas as cargas. Mesmo contendo poros que poderiam facilitar à passagem d'água a zeólita tornou-se uma barreira à passagem do vapor dentro da matriz. Isto pode ser explicado pelo fato da zeólita possuir uma densidade de carga dentro da sua rede cristalina, devido aos átomos de alumínio, o que faz com que a água absorvida fique retida em seu interior. Sendo assim a pressão gerada no experimento não é suficientemente alta para fazer com que a água passe através da sua rede de canais.

Esse comportamento é inferior ao observado na literatura quando são utilizadas argilas montmoriloníticas como modificantes. Ning et al. ${ }^{[13,19]}$ observaram redução de até $60 \%$ no valor total da permeabilidade no sistema TPS/argila, porém observaram tendência à estabilização destes valores em cargas próximas a 10\%. Segundo os autores, o efeito de redução de permeabilidade se deve ao aumento do número de barreiras de passagem de gases, pela esfoliação do

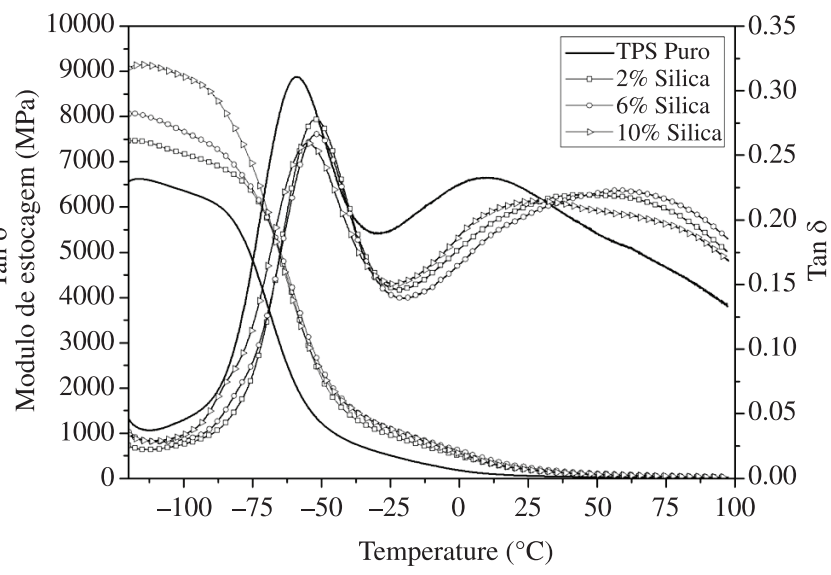




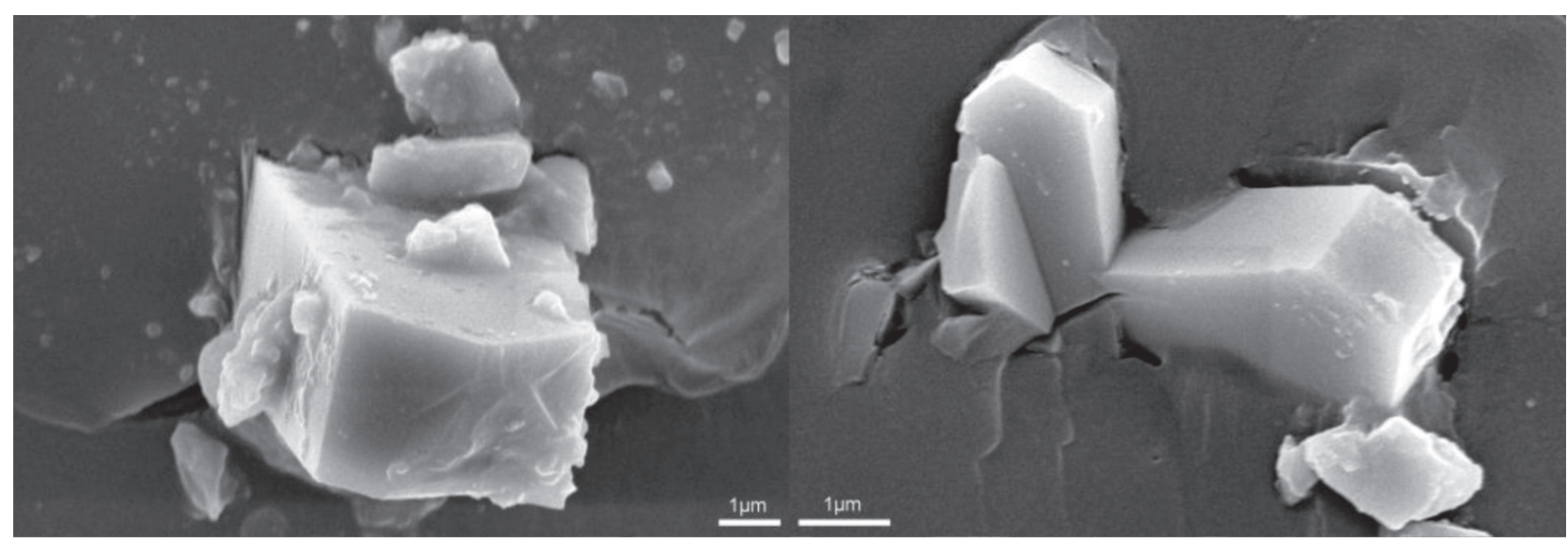

(a)

(b)

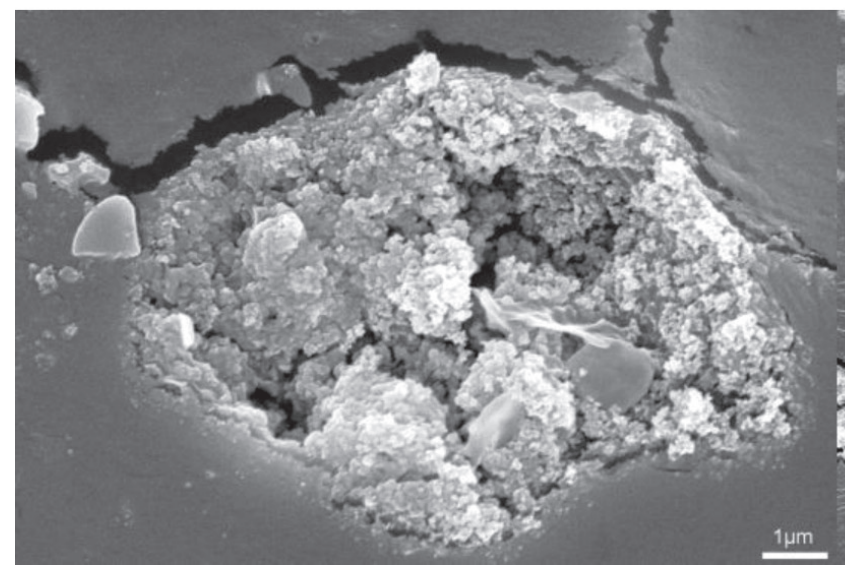

(c)

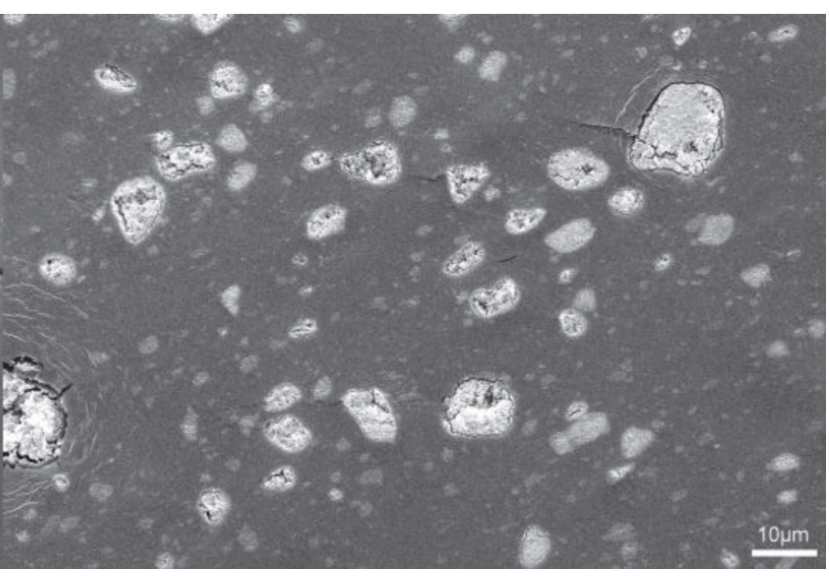

(d)

Figura 6. Micrografia das secções transversais dos corpos de prova, zeólita A e B, sílica C e D.

Tabela 2. Permeabilidade a vapor d'água, valores médios.

\begin{tabular}{|c|c|c|c|}
\hline \multirow{2}{*}{ Composição } & \multicolumn{3}{|c|}{ Permeabilidade a vapor d'água $\left(P_{w}\right)$} \\
\hline & $10^{-5} \cdot \mathbf{g} \cdot \mathbf{m}^{-1} \cdot \mathrm{dia}^{-1} \cdot \mathbf{P a}^{-1}$ & $\boldsymbol{P}_{w} \boldsymbol{P}_{w, T P S}(\%)$ & $r^{2}$ \\
\hline TPS - Puro & $6,77 \pm 0,2$ & 100 & 0,999 \\
\hline $2 \% \mathrm{ZSM}-5$ & $6,92 \pm 0,2$ & 102 & 0,998 \\
\hline $4 \% \mathrm{ZSM}-5$ & $6,38 \pm 0,3$ & 94 & 0,999 \\
\hline $6 \%$ ZSM-5 & $5,97 \pm 0,1$ & 88 & 0,999 \\
\hline $8 \%$ ZSM-5 & $5,61 \pm 0,1$ & 82 & 0,999 \\
\hline $10 \%$ ZSM-5 & $5,89 \pm 0,2$ & 87 & 0,998 \\
\hline $2 \%$ sílica & $5,89 \pm 0,5$ & 87 & 0,998 \\
\hline 4\% sílica & $5,44 \pm 0,4$ & 80 & 0,998 \\
\hline $6 \%$ sílica & $6,25 \pm 0,4$ & 92 & 0,998 \\
\hline $8 \%$ sílica & $5,28 \pm 0,2$ & 77 & 0,998 \\
\hline $10 \%$ silica & $5,85 \pm 0,1$ & 86 & 0,999 \\
\hline
\end{tabular}

argilomineral no polímero. Assim, apesar da alta área superficial dos materiais utilizados neste trabalho, sua estrutura aglomerada rígida impede que estas sejam altamente dispersas na matriz, diminuindo seu potencial de redução da permeabilidade. No entanto, deve-se notar que a presença de um efeito de redução de permeabilidade evidência o potencial destas cargas se trabalhadas em condições mais dispersas e mais próximas de dimensões nanométricas.

\section{Conclusão}

Os resultados demonstraram que a introdução de cargas inorgânicas de alta área superficial aumentou as propriedades mecânicas do TPS e reduziu a permeabilidade a vapor d'água, porém em níveis inferiores aos obtidos na literatura para nanoargilas. Observou-se que a dispersão da carga teve maior influência na redução da permeabilidade que a área superficial total do material, observado na sílica coloidal provavelmente pelo aumento de barreiras difusionais ao longo do caminho de difusão. Estes resultados permitem estabelecer um novo parâmetro de comparação para o uso destes materiais de alta área superficial como cargas em compósitos de matriz biodegradável, e devem fornecer base para estudos futuros.

\section{Agradecimentos}

A CAPES pela bolsa concedida, à FINEP, CNPq, FAPESP e Rede Agronano - Embrapa.

\section{Referências Bibliográficas}

1. Amass, W.; Amass, A. \& Tighe, B. - Polym. Int., 47, p.89 (1998). http://dx.doi.org/10.1002/(SICI)1097-0126(1998100)47:2<89::AIDPI86>3.0.CO;2-F

2. Regiani, A. M.; Curvelo, A. A. S.; Gandini, A. \& Pawlicka, A. - Mol. Cryst. Liq. Cryst. Sci. Technol., Sect. A., 353, p.181 (2000).

3. Osman, Z.; Ibrahim, Z. A. \& Arof, A. K. - Carbohydr. Polym., 44, p.167 (2001). http://dx.doi.org/10.1016/S0144-8617(00)00236-8

4. Lorcks, J. - Polym. Degrad. Stab., 59, p.245 (1998).

5. Avérous, L. - J. Macromol. Sci., Polym. Rev., C24, p.231 (2004).

6. Corradini, E.; Carvalho, A. J. F.; Curvelo, A. A. S.; Agnelli, J. A. M. \& Mattoso, L. H. C. - Mater. Res., 10, p.227 (2007). 
7. Kojima, Y.; Usuki, A.; Kawsumi, M.; Okada, A.; Fukushima, Y.; Kurauchi, T. \& Kamigaito, O. - J. Mater. Res., 8, p.1185 (1993). http:// dx.doi.org/10.1557/JMR.1993.1185

8. Lan, T. \& Pinnavaia, T. J. - Chem. Mater., 6, p.2216 (1994). http:// dx.doi.org/10.1021/cm00048a006

9. Sozer, N. \& Kokini, J. L. - Trends Biotechnol., 27, p.82 (2009). PMid:19135747. http://dx.doi.org/10.1016/j.tibtech.2008.10.010

10. Corradini, E.; Imam S. H ; Agnelli, J. A. M. \& Mattosos L. H. C - J. Polym. Environ., 17, p.1 (2009). http://dx.doi.org/10.1007/s10924009-0115-1

11. Amalvy, J. I.; Percy, M. J.; Aarmes, S. P.; Leite, C. A. \& Galembeck, F. - Langmuir., 21, p.1175 (2005). PMid:15697257. http://dx.doi. org/10.1021/la047535g

12. Le Corre, D.; Bras, J. \& Dufresne, A. - Biomacromolecules., 11, p.1139 (2010). PMid:20405913. http://dx.doi.org/10.1021/bm901428y

13. Ning, W.; Xingxiang, Z.; Na, H. \& Haihui, L. - J. Compos. Mater, 44, p.27 (2010). http://dx.doi.org/10.1177/0021998309345350

14. Van Grieken, R.; Sotelo, J. L.; Menéndez, J. M. \& Melero, J. A. - Microporous Mesoporous Mater., 39, p.135 (2000). http://dx.doi. org/10.1016/S1387-1811(00)00190-6

15. Aguado, J.; Serrano, D. P.; Escola, J. M. \& Rodriguez, J. M. - Microporous Mesoporous Mater., 75, p.41 (2004). http://dx.doi. org/10.1016/j.micromeso.2004.06.027

16. Plotegher, F. \& Ribeiro, C. - "Síntese e caracterização da zeólita ZSM5 para uso em sistemas de adsorção química", Comunicado Técnico, Embrapa, São Carlos (2009).

17. Corradini, E.; Lotti, C.; Medeiros, E. S.; Carvalho, A. J. F.; Curvelo, A. A. S. \& Mattoso, L. H. C. - Polímeros, 15, p.268 (2005).
18. American Society for Testing and Materials - ASTM. - "E96/E96M05: Standard test method for water vapor transmission of materials", Pennsylvania (2005).

19. Ning, W.; Xingxiang, Z.; Na, H. \& Shihe, B. - Carbohydr. Polym., 76, p.68 (2009).

20. Treacy, M. M. J. \& Higgins, J. B. - "Collection of the simulated XDR powder patterns for zeolites", Elsevier, New York (2001).

21. Baerlocher, C.; McCusker, L. B.; \& Olson, D. H. - "Atlas of Zeolite Framework Types", Elsevier, New York (2007).

22. Cheng, Y.; Liao, R. H.; Li, J. S.; Sun, X. Y. \& Wang, L. J. - J. Mater. Process. Technol., 206, p.445 (2008). http://dx.doi.org/10.1016/j. jmatprotec.2007.12.054

23. Mintova, S.; Valtchev, V.; Vulcheva, E. \& Veleva, S. - Zeolites, 12, p.210 (1992). http://dx.doi.org/10.1016/0144-2449(92)90086-5

24. Li, Q.; Wang, Z.; Hedlund, J.; Creaser, D.; Zhang, H.; Zou, X. \& Jan Bons, A. - Microporous Mesoporous Mater., 78, p.1 (2005). http:// dx.doi.org/10.1016/j.micromeso.2004.09.010

25. Mignoni, M. L.; Detoni, C.; Pergher, S. B. C. - Quím. Nova, 30, p.45 (2007). http://dx.doi.org/10.1590/S0100-40422007000100010

26. American Society for Testing and Materials - ASTM. - "E-104-85: Standard practice for maintaining constant humidity by means of aqueous solutions", Pennsylvania (1991).

27. Avérous, L.; Moro, L.; Dole, P. \& Fringant, C. - Polymer, 41, p.4157 (2000). 